\title{
Dithia-diaza[n.2] metacyclophan-ene
}

\author{
Hans-Friedrich Grützmacher* und Jürgen Schmiegel
}

Fakultät für Chemie der Universität Bielefeld,

Postfach 8640, Universitätsstraße, D - 4800 Bielefeld

Eingegangen am 23. März 1989

Key Words: Dithia-diaza[n.2]metacyclophan-enes / Metacyclophanes / Photoisomerization

Dithia-diaza[n.2]metacyclophan-ene 5a-f mit $\mathbf{n}=6,7,8,10$, 12 und 16 werden nach dem „Rigid-Group“-Prinzip durch Kupplung von 1,2-Bis[3-(brommethyl)phenyl]-4,4-dimethyl-3,5-pyrazolidindion (2) mit entsprechenden 1, $\omega$-Alkandithiolen 3a-f unter Anwendung der Verdünnungsmethode und anschließende $\mathrm{Ab}$ spaltung der Dimethylmalonyl-Schutzgruppe hergestellt. ${ }^{1} \mathrm{H}$ NMR-Spektren beweisen für $5 \mathbf{a}(\mathbf{n}=6)$ und für $5 \mathbf{b}(\mathbf{n}=7)$ eine cis-Azokonfiguration mit schneller anti/anti'-Konformationsumwandlung, für 5d-f mit längeren Verbindungsketten eine transAzokonfiguration. Alle trans-Isomere lassen sich photochemisch bei $\lambda=369 \mathrm{~nm}$ quantitativ in die cis-Isomeren umwandeln. cis5 b liefert unter diesen Bedingungen ein photostationäres cis/transGemisch mit ca. $60 \%$ des erheblich gespannten trans-5b, während 5a weder thermisch noch photochemisch in das trans-Isomere übergeführt werden kann.

Kürzlich berichteten wir über Synthese und Eigenschaften von Dithia-diaza[n.2]paracyclophanen ". Diese Heteracyclophane besitzen eine Azogruppe als kurze Brücke der paraverknüpften Benzolringe und lassen sich daher auch als [n]Paraazobenzolophane beschreiben. Bei hinreichend kurzer Verbindungskette kann im Diaza-paracyclophan nur eine cis-Azobenzolkonfiguration vorliegen, bei längerer Kette ist sowohl eine cis- als auch eine trans-Anordnung möglich. Die cis/trans-Photoisomerisierung von Azobenzolen ist gut bekannt ${ }^{2}$. Die Übertragung der Isomerisierung auf die Dithia-diaza[n.2]paracyclophane führt zu einfachen photosensitiven Phanen, die ihre Molekülgestalt definiert bei Belichtung in Abhängigkeit von der Wellenlänge ändern. Besonders bei mittleren Längen der Kette konnten dabei interessante konformative Effekte NMR-spektroskopisch beobachtet werden ${ }^{11}$.

Metacyclophane können sowohl eine syn-Konformation mit übereinanderliegenden Benzolringen als auch eine stufenförmige anti-Konformation einnehmen. [n.2]Metacyclophane $(n>3)$ sind im allgemeinen konformativ beweglich und bevorzugen die anti-Konformation ${ }^{31}$. Für das 2-Thia-10,11-diaza[3.2]metacyclophan-10-en konnten wir jedoch zeigen ${ }^{4}, \mathrm{daB}$ in $\mathrm{CDCl}_{3}$ syn- und anti-Konformation am Gleichgewicht mit ähnlichen Konzentrationen beteiligt sind, und $\mathrm{da} B$ in $\left[\mathrm{D}_{6}\right]$-Aceton und anderen polaren Lösungsmitteln die syn-Konformation durch spezifische Solvatation oder Anlagerung eines Lösungsmittelmoleküls bevorzugt wird. Für Dithia-diaza[n.2]metacyclophane mit langer Verbindungskette sind neben Stereoisomeren mit cis/ trans-Konfiguration an der Azobrücke eine größere Vielfalt von Konformationen möglich, wobei ebenfalls photosensi-

\section{Dithia-diaza[n.2]metacyclophan-enes}

Dithia-diaza[n.2]metacyclophan-enes $5 \mathrm{a}-\mathrm{f}$ with $\mathrm{n}=6,7,8,10$, 12 , and 16 have been prepared by using the rigid-group principle and the high-dilution technique by coupling of 1,2-bis[3-(bromomethyl)phenyl]-4,4-dimethyl-3,5-pyrazolidindione (2) with appropriate 1, $\omega$-alkanedithiols $3 \mathrm{a}-\mathrm{f}$ and subsequent removal of the dimethylmalonyl protecting group. The ' $H$-NMR spectra establish a cis-azoconfiguration and a fast anti/anti'-conformational change for $5 \mathrm{a}(\mathrm{n}=6)$ and $5 \mathrm{~b}(\mathrm{n}=7)$ and a trans-azoconfiguration for $5 d-f$ containing larger bridges. trans-5d-f isomerize photochemically at $\lambda=369 \mathrm{~nm}$ quantitatively into the cis-isomers. cis$5 \mathbf{b}$ yields under the same conditions a photostationary cis/trans mixture containing ca. $60 \%$ of the considerably strained trans$\mathbf{5 b}$, while $\mathbf{5 a}$ is neither thermically nor photochemically transformed into the cis isomer.

tive Cyclophane vorliegen und zudem Lösungsmitteleffekte auf das Konformationsgleichgewicht möglich scheinen.

Im Rahmen einer verbesserten Synthese des 2-Thia-10,11diaza[3,2]metacyclophan-10-ens ${ }^{\text {S) }}$ nach dem „Rigid-Group“Prinzip $^{6)}$ wurde 1,2-Bis[3-(brommethyl)phenyl]-4,4-dimethyl-3,5-pyrazolidindion (2) als Vorstufe verwendet. 2 enthält die Hydrazobenzoleinheit in einer relativ starren cisAnordnung, so da $B$ die reaktiven Brommethylsubstituenten in den meta-Positionen für den Cyclophanringschluß sterisch günstig angeordnet sind. 2 eignet sich damit gut zum Aufbau von Dithia-diaza[n.2]metacyclophan-enen. Wir berichten in dieser Arbeit über Synthese und spektroskopische Eigenschaften dieser Diaza-metacyclophane.

\section{Synthesen}

2 kann durch direkte Photobromierung des 4,4-Dimethyl1,2-bis(3-methylphenyl)-3,5-pyrazolidindions (1) mit $\mathrm{N}$ Bromsuccinimid (NBS) bei sorgfältiger Reaktionsführung mit $46 \%$ Ausbeute erhalten werden (Schema 1). Das Pyrazolidinderivat 1 ist durch Kondensation von Dimethylmalonsäuredichlorid mit $m$-Hydrazotoluol unter speziellen Bedingungen gut zugänglich.

Die Ausbeute beim nachfolgenden Ringschluß zu den Diaza-metacyclophanen 4a-f durch Umsetzung mit 1, $\omega$ Alkandithiolen 3a-f hängt erheblich von der Reinheit des Dibromids $2 \mathrm{ab}$. Es ist deswegen eine Abtrennung von unterund überbromierten Verunreinigungen durch Chromatographie an Mitteldrucksäulen (Eluens Petrolether/Essigsäure-ethylester, 9:1) unbedingt notwendig. Das Dibromid 2 ist ebenso reaktiv wie das para-Isomere ${ }^{\mathrm{l}}$ und läßt sich 
Schema 1

$$
\prod_{0}^{0} \mathrm{Cl}^{\mathrm{Cl}}
$$<smiles>Cc1cccc(NNc2cccc(C)c2)c1</smiles><smiles>Cc1cccc(N2C(=O)C(C)(C)C(=O)N2c2cccc(C)c2)c1</smiles>
1

$1 \stackrel{2 \text { NBS }}{\stackrel{\text { CCI }}{\longrightarrow}}$<smiles>CC1(C)C(=O)N(c2cccc(CBr)c2)N(c2cccc(CBr)c2)C1=O</smiles>

2

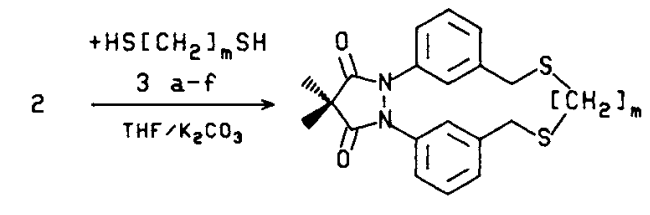

$4 a-f$
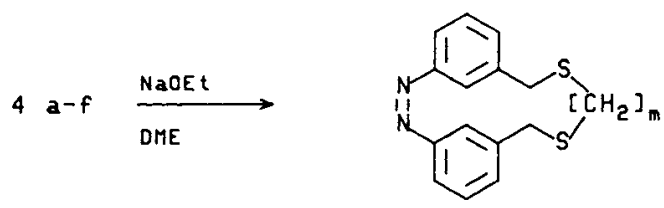

$5 a-f$

\begin{tabular}{c|cccccc} 
& a & b & c & d & e & f \\
\hline $\mathrm{m}$ & 2 & 3 & 4 & 6 & 8 & 12
\end{tabular}

unter Anwendung des Verdünnungsprinzips glatt mit 3a-f in Tetrahydrofuran (THF) oder Benzol in Anwesenheit von trockenem $\mathrm{K}_{2} \mathrm{CO}_{3}$ phasentransfer-katalytisch zu den $N, N^{\prime}$-geschützten Dithia-diaza[n.2]metacyclophan-Derivaten $4 \mathbf{a}-\mathbf{f}$ umsetzen.

Die $N, N^{\prime}$-geschützten Diazacyclophane 4 werden nach Säulenchromatographie als farblose Verbindungen mit Ausbeuten von $36 \%(4 a)$ bis $64 \%$ (4f) erhalten. Anders als bei den Diaza-paracyclophan-Derivaten" hat die Größe des entstehenden Ringes, sorgfältige Reaktionsführung vorausgesetzt, keinen Einfluß auf die Ausbeute. Offensichtlich ist die Ringspannung bei allen $4 a-f$ wenig unterschiedlich und wegen der Flexibilität des Metacyclophangerüstes gering. Die Freisetzung der Dithia-diaza[n.2]metacyclophan-ene 5a-f durch Abspalten der Dimethylmalonyl-Schutzgruppe und schonende Oxidation der zunächst entstehenden Metahydrazobenzolophane gelingt durch Behandeln mit über schüssigem Natriumethanolat in 1,2-Dimethoxyethan (DME) bei Raumtemperatur mit guten Ausbeuten. Auch bei diesem Reaktionsschritt macht sich anders als bei den analogen Diaza-paracyclophanen ${ }^{13}$ kein durch die Ringgröße bestimmter Stabilitätsunterschied bei den Ausbeuten bemerkbar. Ein (oxidativer) Ringschlu $B$ zu 9,10-Diazaphenanthren-Derivaten, der beim 2-Thia-10,11-diaza[3.2]metacyclophan-10-en unter den verschiedensten Reaktionsbedin- gungen so außerordentlich leicht erfolgt ${ }^{4}$, wurde ebenfalls nicht beobachtet.

Tab. 1. Ausbeuten und Gleichgewichtsverhältnisse der cis/transIsomeren $^{\text {a) }} \mathbf{5}$

\begin{tabular}{cccccc}
\hline $\begin{array}{c}\text { Verbin- } \\
\text { dung }\end{array}$ & $\begin{array}{c}\text { Ausb. } \\
(\%)\end{array}$ & $\begin{array}{c}\text { cis/trans- } \\
\text { Verhältnis } \\
(\%)\end{array}$ & $\begin{array}{c}\text { Verbin- } \\
\text { dung }\end{array}$ & $\begin{array}{c}\text { Ausb. } \\
(\%)\end{array}$ & $\begin{array}{c}\text { cis/trans- } \\
\text { Verhältnis } \\
(\%)\end{array}$ \\
\hline 5a & 51 & $100: 0$ & $\mathbf{5 d}$ & 75 & $10: 90$ \\
5b & 63 & $70: 30$ & $\mathbf{5 e}$ & 66 & $<1: 99$ \\
$\mathbf{5 c}$ & 79 & $40: 60$ & $\mathbf{5 f}$ & 72 & $<1: 99$
\end{tabular}

a) Bei Raumtemperatur und diffusem Tageslicht.

Die Dithia-diaza[n.2]metacyclophan-ene 5a-f bilden orangerote bis rote Kristalle, wobei mit steigendem $\mathbf{n}$ eine deutliche Farbvertiefung beobachtet wird. Die Diazametacyclophane $\mathbf{5} \mathbf{b}-\mathbf{d}(\mathrm{n}=7,8,10)$ fallen als cis/trans-Gemische an, die sich dünnschichtchromatographisch in das gelbliche cis-Isomere mit kleinerem $R_{\mathrm{r}}$ Wert und das tiefrote transIsomere trennen lassen. Nach der Trennung stellt sich jedoch bei Raumtemperatur und bei diffusem Tageslicht schnell die Gleichgewichtsmischung wieder ein.

Die Massenspektren der $N, N^{\prime}$-geschützten Diazacyclophane $4 \mathbf{a}-\mathbf{f}$ zeigen jeweils ein intensives Signal für $\mathbf{M}^{+\bullet}$; mit der Ausnahme 4d stellt dies den Basispeak des Spektrums dar. Sehr intensive $\mathrm{M}^{+}$sind bei Cyclophanen häufig zu beobachten. Das typische Fragmention von $4 \mathbf{a}-\mathbf{f}$ bei $m / z=306 / 305$ kommt durch die Abspaltung der eingebauten Dithiabrücke zustande; Abspaltung der Schutzgruppe daraus liefert das Ion $m / z=207$. Weitere typische Ionen für die mit einem $N, N^{\prime}$-Dimethylmalonylrest geschützten Cyclophane sind $m / z=132$ und 146 .

Die freien Dithia-diaza-metacyclophane 5a-f liefern im EI-Massenspektrum ebenfalls intensive Peaks für die Molekül-Ionen, deren Intensität mit größerer Kettenlänge zunimmt und bei 5e und 5f 100\% erreicht. Der Basispeak der kleineren Cyclophane entsteht durch ein Ion $m / z=180 \mathrm{mit}$ der Masse des Dihydrophenanthrens. Im oberen Massenbereich beobachtet man ferner ein intensives Signal bei $m / z=211$. Die Massenfeinbestimmung ergibt, da $\beta$ dieses Ion durch den Verlust der $\mathrm{N}_{2}$-Brücke und entsprechender (C,H,S)-Fragmente aus der zweiten Brücke entsteht. Der Verlust von $\mathrm{N}_{2}$ ist eine typische massenspektrometrische Fragmentierung für aromatische Azoverbindungen. Im unteren Massenbereich spiegeln die Fragment-Ionen $\mathrm{C}_{7} \mathrm{H}_{6}^{+}$ und $\mathrm{C}_{7} \mathrm{H}_{5}^{+}(m / z=90$ bzw. 89) den Aufbau der Moleküle aus $\mathrm{zwei} \mathrm{C}_{7} \mathrm{H}_{6}$-Einheiten gut wider.

Die UV-Spektren zeigen im Gegensatz zu den Dithiadiaza[n.2]paracyclophanen ${ }^{11}$ keine großen Unterschiede für die einzelnen Isomere. Dies entspricht der Erwartung, da $B$ alle Metacyclophane 5a-f wenig gespannte Verbindungen sind.

\section{${ }^{1}$ H-NMR-Spektroskopie}

Zur Zuordnung der cis/trans-Konfiguration an der Azogruppe der Dithia-diaza[n.2]metacyclophan-ene 5a-f und 
zur Untersuchung der Konformationen eignet sich die ${ }^{1} \mathrm{H}-$ NMR-Spektroskopie gut. trans-meta-Azotoluol liefert im ${ }^{1} \mathrm{H}-\mathrm{NMR}$-Spektrum $\left(\mathrm{CDCl}_{3}\right)$ das erwartete Signalmuster für die Protonen der aromatischen Ringe bei $\delta>7.2$, wobei das Singulett für das isolierte Proton zwischen Azo- und Methylsubstituent (im folgenden mit $\mathrm{H}_{\mathrm{i}}$ bezeichnet) bei $\delta=$ 7.71 erscheint. Für die Methylsubstituenten wird ein Singulett bei $\delta=2.46$ beobachtet. Für das cis-meta-Azotoluol, das im Gemisch mit dem trans-Isomeren durch Bestrahlen der $\mathrm{CDCl}_{3}$-Lösung erhalten wird, erscheinen die Signale der Protonen an den aromatischen Ringen bei $\delta<7.2 \mathrm{mit}$ dem Singulett für $\mathrm{H}_{\mathrm{i}}$ bei $\delta=6.78$, und die Methylsubstituenten liefern ein ebenfalls hochfeldverschobenes Singulett bei $\delta=$ 2.28. Schließlich kann aufgrund des 'H-NMR-Spektrums des 2-Thia-10,11-diaza[3.2]metacyclophan-10-ens ${ }^{4)}$ erwartet werden, daß auch bei den größeren Cyclophanen $5 \mathbf{a}-\mathbf{f}$ sich die syn- und anti-Konformationen durch eine besonders ausgeprägte Hochfeldverschiebung des Signals von $\mathrm{H}_{i}$ in der anti-Form unterscheiden.

Dreiding-Modelle zeigen, daß eine planare Azobenzoleinheit spannungsfrei erst beim Dithia-diaza[10.2]metacyclophan-en 5d $(n=10)$ möglich sein sollte und insbesondere das Dithia-diaza[6.2]metacyclophan-en 5a nur eine cis-Azokonfiguration einnehmen kann. Das ${ }^{1} \mathrm{H}$-NMR-Spektrum von $5 \mathbf{a}\left(\left[\mathrm{D}_{8}\right]\right.$ Toluol, Tab. 2) zeigt dann auch das für die cisForm erwartete hochfeldverschobene Multiplett der drei benachbarten Protonen der aromatischen Ringe bei $\delta=6.92$, während das Singulett von $\mathrm{H}_{\mathrm{i}}$ bei $\delta=5.83$ besonders auffällig hochfeldverschoben ist. 5a liegt demnach in einer cisanti-Konformation vor; Hinweise auf die cis-syn-Konformation wie beim 2-Thia-10,11-diaza[3.2]metacyclophan-10$\mathrm{en}^{4)}$ sind nicht vorhanden. Für die benzylischen Protonen von 5a wird im NMR-Spektrum $\left(\mathrm{CDCl}_{3}\right)$ bis $336 \mathrm{~K}$ stets ein scharfes Singulett beobachtet. Für die anti-Konformation eines Metacyclophans bedeutet dies einen schnellen Wechsel auch bei tiefen Temperaturen zwischen der antiund der anti'-Konfiguration.

Das ${ }^{1} \mathrm{H}$-NMR-Spektrum beweist auch für das Dithia-diaza[7,2]metacyclophan-en $\mathbf{5 b}$ eine Bevorzugung der cis-antiAnordnung (Tab. 2). Die längere Kette erlaubt jedoch ein stärkeres Auseinanderrücken der Benzolringe, so daß die Hochfeldverschiebung von $\mathrm{H}_{\mathrm{i}}(\delta=6.12)$ geringer ausfällt als bei 5a. Entgegen der Erwartung sind jedoch im NMRSpektrum von $\mathbf{5 b}$ auch schon die Signale des trans-Isomeren vorhanden; und eine Auswertung der Integrale ergibt einen Anteil der trans-Form von ca. 30\%. Ebenso erweist sich 5c aufgrund des ${ }^{1} \mathrm{H}-\mathrm{NMR}$-Spektrums als ein Gemisch der cis/ trans-Isomeren, in dem jetzt die trans-Form sogar mit einem Anteil von ca. $60 \%$ überwiegt. In der trans-Form absorbiert $\mathrm{H}_{\mathrm{i}}$ bei $\delta=7.83$ als Singulett mit Feinstruktur (Tab. 2). Das Dublett bei $\delta=7.66\left(J_{\mathrm{AB}}=8 \mathrm{~Hz}\right)$ kann dem aromatischen Proton $\mathrm{H}_{\mathrm{A}}$ in Nachbarschaft zur Azogruppe zugeordnet werden. Das Triplett von $\mathrm{H}_{\mathrm{B}}$ erscheint bei $\delta=7.10\left(J_{\mathrm{AB}}=\right.$ $J_{\mathrm{BC}}=8 \mathrm{~Hz}$ ), während $\mathrm{H}_{\mathrm{C}}$, benachbart zur zweiten Brücke im Molekül, bei $\delta=6.86$ absorbiert und ebenfalls zu einem Dublett mit Feinstruktur aufspaltet. Die benzylischen Protonen ergeben ein Singulett bei $\delta=3.52$, während die Protonen der zum S-Atom $\alpha$-ständigen $\mathrm{CH}_{2}$-Gruppe der zweiten Brücke ein charakteristisches Multiplett bei $\delta=2.20$ liefern. Auch die Signalform des Multipletts der Protonen der zum S-Atom $\beta$-ständigen $\mathrm{CH}_{2}$-Gruppe bei $\delta=1.42$ ist charakteristisch für die trans-Form. In der cis-Form absorbiert $\mathrm{H}_{\mathrm{i}}$ als Singulett hochfeldverschoben bei $\delta=6.25$. Die Signale für $H_{A}$ und $H_{B}$ sind ebenfalls hochfeldverschoben, das von $\mathrm{H}_{C}$ dagegen nicht. Die Absorptionen von $\mathrm{H}_{\mathrm{A}}$ und $\mathrm{H}_{\mathrm{C}}$ überlagern sich bei $\delta=6.82$, während $\mathrm{H}_{\mathrm{B}}$ ein nicht gut aufgelöstes Triplett bei $\delta=6.62$ liefert. Die benzylischen Protonen erscheinen nun bei $\delta=3.10$ als Singulett, und die Protonen der $\mathrm{CH}_{2}$-Gruppen zwischen den S-Atomen der Dithiabrücke zeigen charakteristische, hochfeldverschobene Multipletts bei $\delta=1.76$ und 1.11. Die unterschiedlichen chemischen Verschiebungen für die Protonen beider Isomeren sind so gut zu erkennen.

Für die Dithia-diaza[10.2]-, -[12.2]- und -[16.2]metacyclophan-ene $5 \mathbf{d}-\mathbf{f}$ werden in $\left[\mathrm{D}_{8}\right]$ Toluol nur die ${ }^{1} \mathrm{H}$ NMR-Spektren der trans-Isomeren gefunden. Diese zeichnen sich durch ein $\mathrm{H}_{\mathrm{i}}$-Signal bei besonders tiefem Feld bei $\delta=7.96$ (5d), 7.90 (5e) und 7.93. (5f) aus (Tab. 2).

Die Gleichgewichtskonzentrationen der Konformeren von $\mathbf{5 a}-\mathbf{f}$ werden durch das Lösungsmittel nicht erkennbar

Tab. 2. ${ }^{1} \mathrm{H}$-NMR-Daten ( $\delta$-Werte) der Dithia-diaza-metacyclophane 5 in $\left[\mathrm{D}_{8}\right]$ Toluol [ppm]

\begin{tabular}{|c|c|c|c|c|c|c|c|c|c|}
\hline & $\mathrm{H}_{\mathrm{A}}$ & $\mathrm{H}_{\mathrm{B}}$ & $\mathbf{H}_{\mathrm{C}}$ & $\mathrm{H}_{\mathrm{i}}$ & $\mathrm{H}_{\text {Benzyl }}$ & & & $\mathrm{H}_{\left(\mathrm{CH}_{2}\right)}$ & \\
\hline 5a, cis & & $6.92 \mathrm{~m}$ & & $5.83 \mathrm{~s}$ & $2.90 \mathrm{~s}$ & $1.84 \mathrm{~s}$ & & & \\
\hline $\mathbf{5 b}$, cis & $6.61 \mathrm{~d}$ & \multicolumn{2}{|c|}{$6.98 \mathrm{~m}$} & $6.12 \mathrm{~s}$ & $2.98 \mathrm{~s}$ & $1.85 \mathrm{t}$ & $1.14 \mathrm{~m}$ & & \\
\hline trans & $7.62 \mathrm{~d}$ & $7.06 \mathrm{t}$ & $6.74 \mathrm{~d}$ & $7.45 \mathrm{~s}$ & $3.38 \mathrm{~s}$ & $1.95 \mathrm{~m}$ & $1.61 \mathrm{~m}$ & & \\
\hline $5 \mathbf{c}, c i s$ & $6.82 \mathrm{dd}$ & $6.62 \mathrm{t}$ & $6.82 \mathrm{dd}$ & $6.25 \mathrm{~s}$ & $3.10 \mathrm{~s}$ & $1.76 \mathrm{~m}$ & $1.11 \mathrm{~m}$ & & \\
\hline trans & $7.66 \mathrm{~d}$ & $7.10 \mathrm{t}$ & $6.86 \mathrm{~d}$ & $7.83 \mathrm{~s}$ & $3.52 \mathrm{~s}$ & $2.20 \mathrm{~m}$ & $1.41 \mathrm{~m}$ & & \\
\hline $5 \mathrm{~d}, c i s$ & $6.59 \mathrm{~d}$ & $6.80 \mathrm{t}$ & $6.88 \mathrm{t}$ & $6.45 \mathrm{~s}$ & $3.16 \mathrm{~s}$ & $2.01 \mathrm{t}$ & $1.10 \mathrm{~m}$ & & \\
\hline trans & $7.76 \mathrm{~d}$ & $7.14 \mathrm{t}$ & - & $7.96 \mathrm{~s}$ & $3.53 \mathrm{~s}$ & $2.23 \mathrm{t}$ & $1.47 \mathrm{~m}$ & $1.23 \mathrm{~m}$ & \\
\hline $5 \mathbf{e}$, cis & $6.56 \mathrm{~d}$ & $6.86 \mathrm{t}$ & $6.94 \mathrm{~d}$ & $6.68 \mathrm{~s}$ & $3.19 \mathrm{~s}$ & $2.05 \mathrm{t}$ & $1.30 \mathrm{~m}$ & & \\
\hline trans & $7.86 \mathrm{~d}$ & $7.14 \mathrm{t}$ & $7.14 \mathrm{~d}$ & $7.90 \mathrm{~s}$ & $3.48 \mathrm{~s}$ & $2.09 t$ & $1.46 \mathrm{~m}$ & $1.10 \mathrm{~m}$ & \\
\hline $\mathbf{5 f}$, cis & $6.39 \mathrm{~d}$ & $6.75 \mathrm{t}$ & $6.81 \mathrm{~d}$ & $6.78 \mathrm{~s}$ & $3.25 \mathrm{~s}$ & $2.05 \mathrm{~s}$ & $1.10 \mathrm{~m}$ & & \\
\hline $\begin{array}{c}\text { trans } \\
m \text {-Azotoluol }\end{array}$ & $7.85 \mathrm{~d}$ & $7.15 \mathrm{t}$ & $7.26 \mathrm{~d}$ & $7.93 \mathrm{~s}$ & $3.46 \mathrm{~s}$ & $2.22 \mathrm{t}$ & $1.37 \mathrm{~m}$ & $1.16 \mathrm{~m}$ & $1.06 \mathrm{~m}$ \\
\hline cis & $6.52 \mathrm{~d}$ & $7.08 \mathrm{t}$ & $6.95 \mathrm{~d}$ & $6.78 \mathrm{~s}$ & $2.27 \mathrm{~s}$ & & & & \\
\hline trans & $7.73 \mathrm{~d}$ & $7.40 \mathrm{t}$ & $7.29 \mathrm{~d}$ & $7.73 \mathrm{~s}$ & $2.46 \mathrm{~s}$ & & & & \\
\hline
\end{tabular}


Schema 2

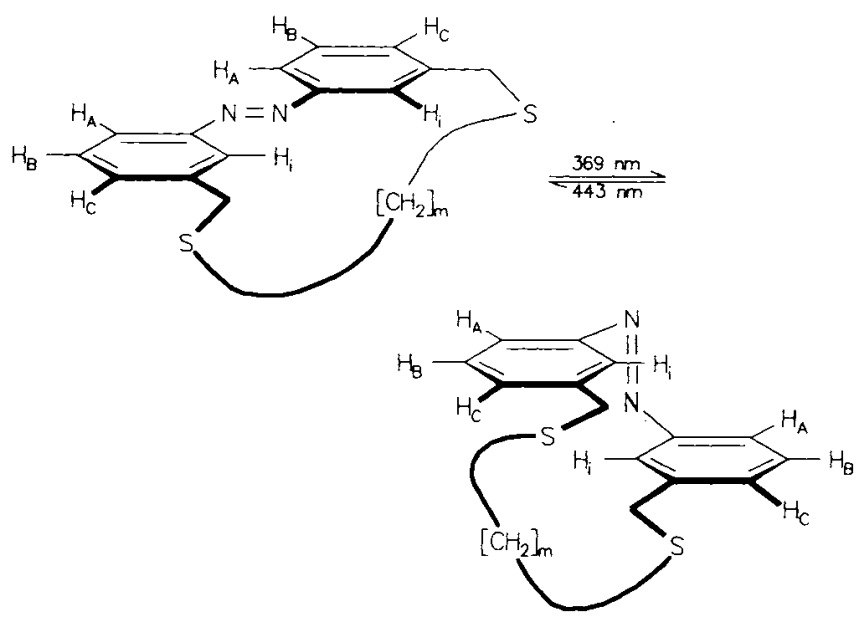

beeinflußt. Die ${ }^{1} \mathrm{H}-\mathrm{NMR}$-Spektren weisen beim Wechsel von $\mathrm{CDCl}_{3}$ zu $\mathrm{CD}_{3} \mathrm{CN}$ oder $\left[\mathrm{D}_{8}\right]$ Toluol nur vergleichsweise geringfügige Veränderungen auf. Die bemerkenswerte Koordination von Lösungsmittelmolekülen, die für das 2-Thia10,11-diaza[3.2]metacyclophan-10-en beobachtet wurde ${ }^{4}$, wiederholt sich also bei den größeren Dithia-diaza-metacyclophanen $5 \mathbf{a}-\mathbf{f}$ nicht. Wie die Dithia-diaza[n.2]paracyclophan-ene ${ }^{1)}$ lassen sich jedoch trans-5c-f durch Bestrahlen bei $\lambda=369 \mathrm{~nm}$ in $\mathrm{CD}_{3} \mathrm{CN}$ oder in $\left[\mathrm{D}_{8}\right]$ Toluol glatt zu den cis-Verbindungen photoisomerisieren (Schema 2). Für cis-5c weist die Lage des $\mathrm{H}_{\mathrm{i}}$-Signals im ' $\mathrm{H}$-NMR-Spektrum bei $\delta=6.25$ noch auf eine cis-anti-Konformation hin; bei cis-5d liegt das Signal für $\mathrm{H}_{\mathrm{i}}$ mit $\delta=6.54$ bereits im Bereich der übrigen aromatischen Protonen. Die aus 5b-f photochemisch erzeugten cis-Isomere lagern sich beim Stehenlassen im Dunkeln wieder völlig in die stabilere transForm um. Bemerkenswert ist, daß cis-5b beim Bestrahlen mit Licht der Wellenlänge $\lambda=443 \mathrm{~nm}$ in $\mathrm{CD}_{3} \mathrm{CN}$ oder in $\left[\mathrm{D}_{8}\right]$ Toluol ein photostationäres cis/trans-Gemisch mit ca. $60 \%$ trans-Anteil liefert, obwohl in diesem Cyclophan eine trans-Azobenzoleinheit nach Dreiding-Modellen spannungsfrei nicht möglich ist und MMPMI-Rechnungen ") für trans-5b eine Spannungsenergie von $120 \mathrm{~kJ} / \mathrm{mol}$ ergeben. Damit verhalten sich die Dithia-diaza[n.2]metacyclophane 5 photochemisch analog ihren para-Isomeren ").

Dem Fonds der Chemischen Industrie sind wir für die finanzielle Unterstützung dankbar. Wir danken Herrn $K$. P. Mester, Universität Bielefeld, für die Messung der 300-MHz-NMR-Spektren und Herrn E. Gärtner, Universität Bielefeld, für die Durchführung der Massenfeinbestimmungen.

\section{Experimenteller Teil}

IR-Spektren: Perkin-Elmer 377. - UV-Spektren: BeckmanSpektralphotometer Modell 25. - Massenspektren: Varian-MAT 311 A. - 'H-NMR-Spektren: Bruker AM 300 und WP 80. Schmelzpunkte (nicht korrigiert): Elektrothermal-SchmelzpunktApparat. - Elementaranalysen: Zentrale Analytik der Fakultät für Chemie der Universität Bielefeld. - Säulenchromatographie: Kieselgel 60, 0.063-0.200 mm (Merck); Mitteldruckchromatographie: Lobar LiChroprep Si 60 (40-63 $\mu \mathrm{m}$; Merck); Flash-Chromatogra- phie: Kieselgel 60, Korngröße kleiner als $0.063 \mathrm{~mm}$ (Merck). Dünnschichtchromatographie: Kieselgel $60 \quad F_{254}$ auf Al-Folie (Merck). - Photoisomerisierungen: $5 \mathrm{mg}$ der jeweiligen Substanz werden in $1 \mathrm{ml}\left[\mathrm{D}_{8}\right]$ Toluol in einem NMR-Rohr bestrahlt; Photolampe Normag TQ 150/Z1; Auswahl der Wellenlängen durch UV-Filter (Schott); $\lambda=443 \mathrm{~nm}\left(\mathrm{~T}_{\max } 41 \%, \mathrm{HW}=14 \mathrm{~nm}\right), \lambda=$ $369 \mathrm{~nm}\left(\mathrm{~T}_{\max } 51 \%, \mathrm{HW}=7,9 \mathrm{~nm}\right)$. - Alle Lösungsmittel wurden destilliert und, wenn nötig, nach gängigen Methoden getrocknet.

4,4-Dimethyl-1,2-bis (3-methylphenyl)-3,5-pyrazolidindion (1): $19.04 \mathrm{~g}(0.11 \mathrm{~mol})$ Dimethylmalonsäuredichlorid in $50 \mathrm{ml}$ trockenem $\mathrm{CHCl}_{3}$ werden bei $-18^{\circ} \mathrm{C}$ unter $\mathrm{N}_{2}$ zu einer Lösung von 40 $\mathrm{ml}$ Pyridin und $0.05 \mathrm{~g}$ 4-(Dimethylamino)pyridin in $100 \mathrm{ml}$ trokkenem $\mathrm{CHCl}_{3}$ getropft. $\mathrm{Zu}$ dieser Lösung gibt man bei $0^{\circ} \mathrm{C}$ unter $\mathrm{N}_{2} 23.9 \mathrm{~g}(0.11 \mathrm{~mol}) m$-Hydrazotoluol in $250 \mathrm{ml}$ trockenem $\mathrm{CHCl}_{3}$. Nach der Zugabe wird die Kühlung entfernt und bis zum Erwärmen auf Raumtemp. gerührt. Dann wird das Lösungsmittel zur Hälfte i. Vak. entfernt, die Lösung 5 mal mit $2 \mathrm{~N} \mathrm{HCl}, 3$ mal mit $\mathrm{H}_{2} \mathrm{O}$ und 2 mal mit 5 proz. $\mathrm{NaHCO}_{3}$-Lösung gewaschen, mit $\mathrm{NaSO}_{4}$ getrocknet und eingeengt. Der Rückstand wird aus Diethylether umkristallisiert; Ausb. $24.3 \mathrm{~g}(64 \%)$ farblose Kristalle (aus Diethylether), Schmp. $104^{\circ} \mathrm{C}, R_{\mathrm{f}}=0.54$ (Toluol/Aceton, 5:1). $-\mathrm{IR}(\mathrm{KBr}): \tilde{v}=$ $1730 \mathrm{~cm}^{-1}(\mathrm{C}=\mathrm{O}) .-{ }^{1} \mathrm{H}-\mathrm{NMR}\left(80 \mathrm{MHz}, \mathrm{CDCl}_{3}\right): \delta=1.49(\mathrm{~s}$, $\left.6 \mathrm{H}, \mathrm{CH}_{3}\right), 2.31\left(\mathrm{~s}, 6 \mathrm{H}\right.$, benzyl. $\left.\mathrm{CH}_{3}\right), 7.18(\mathrm{~m}, 8 \mathrm{H}$, aromat. $\mathrm{H})$. MS: $m / z(\%)=308(100)\left[\mathrm{M}^{+\cdot}\right], 210(7), 146(5), 132(10), 119(27)$.

$$
\begin{array}{ll}
\mathrm{C}_{19} \mathrm{H}_{20} \mathrm{~N}_{2} \mathrm{O}_{2} \text { (308.4) } & \text { Ber. C } 74.0 \mathrm{H} 6.54 \text { N } 9.08 \\
& \text { Gef. C } 74.5 \text { H } 6.54 \text { N } 8.98
\end{array}
$$

1,2-Bis[3-(brommethyl)phenyl]-4,4-dimethyl-3,5-pyrazolidindion (2): $4.00 \mathrm{~g} 1(13 \mathrm{mmol})$ werden in $400 \mathrm{ml}$ trockenem $\mathrm{CCl}_{4}$ gelöst,

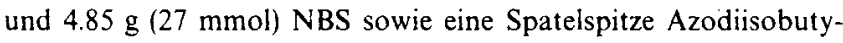
ronitril werden hinzugefügt. Nach 2 stdg. Bestrahlen mit einer $500-$ W-Photolampe bei $40 \mathrm{C}$ Innentemp. wird filtriert, der Filterkuchen mehrfach mit $\mathrm{CCl}_{4}$ gewaschen, die Lösung zur Trockene eingeengt und der Rückstand in wenig Aceton aufgenommen. Die Trennung des Dibromids von der Ausgangssubstanz sowie von Mono- und Tribrom-Derivaten erfolgt durch Mitteldruck-oder Flash-Chromatographie, Eluens Petrolether/Essigester (9:1); Ausb. $3.5 \mathrm{~g}(46 \%)$, farblose, körnige Kristalle (aus dem Eluens), Schmp. $125^{\circ} \mathrm{C}, R_{\mathrm{f}}=$ 0.40 (Petrolether/Essigester, 4:1). - ${ }^{1} \mathrm{H}-\mathrm{NMR}\left(60 \mathrm{MHz}_{2} \mathrm{CDCl}_{3}\right)$ : $\delta=1.50\left(\mathrm{~s}, 6 \mathrm{H}, \mathrm{CH}_{3}\right), 4.38\left(\mathrm{~s}, 4 \mathrm{H}, \mathrm{CH}_{2} \mathrm{Br}\right), 7.33$ (m, $8 \mathrm{H}$, aromat. H). - MS: $m / z(\%)=464 / 466 / 468(100)\left[\mathrm{M}^{+\cdot}\right], 385 / 387(26), 305$ (9), 169/171 (31), $146(18), 132(16), 90(68)$.

$$
\begin{array}{ll}
\mathrm{C}_{19} \mathrm{H}_{18} \mathrm{Br}_{2} \mathrm{~N}_{2} \mathrm{O}_{2} \text { (466.2) } & \text { Ber. C } 49.0 \text { H } 3.89 \text { N } 6.01 \\
& \text { Gef. C 48.7 H } 3.96 \text { N } 5.68
\end{array}
$$

Allgemeine Arbeitsvorschrift zur Darstellung der N.N'-(Dimethylmalonyl)dithia-diaza/n.2/metacyclophane 4a-f: $1.00 \mathrm{~g} 2(2.15$ mmol) und $4.3 \mathrm{mmol}$ des entsprechenden 1, $\omega$-Alkandithiols $3 \mathbf{a}-\mathbf{f}$ werden in $200 \mathrm{ml}$ trockenem THF gelöst und in einer Verdünnungsapparatur nach Vögtle ${ }^{8)}$ während $50 \mathrm{~h}$ zu einer Suspension aus $6.00 \mathrm{~g} \mathrm{~K}_{2} \mathrm{CO}_{3}(43 \mathrm{mmol})$ und $100 \mathrm{mg} \mathrm{18-Krone-6}$ in 21 siedendem THF unter kräftigem Rühren und unter $\mathrm{N}_{2}$ getropft. Die Reaktion wird dünnschichtchromatographisch verfolgt. Nach Beendigung der Reaktion wird zur Trockene eingeengt, der Rückstand mehrfach mit Essigester extrahiert und der Extrakt filtriert. Die Reinigung erfolgt durch Flash-Chromatographie (Eluens Petrolether/Essigester, 2:1).

13,14-(Dimethylmalonyl-2,5-dithia-13,14-diaza/6.2Jmetacyclophan (4a): Ausb. $290 \mathrm{mg}$ (36\%) farblose Nadeln (aus Essigester), Schmp. $156 \mathrm{C}, R_{\mathrm{f}}=0.63$ (Petrolether/Essigester, 2:1). = ${ }^{1} \mathrm{H}-\mathrm{NMR}$ $\left(300 \mathrm{MHz}, \mathrm{CD}_{2} \mathrm{Cl}_{2}\right): \delta=1.51\left(\mathrm{~s}, 6 \mathrm{H}, \mathrm{CH}_{3}\right), 2.18\left(\mathrm{~s}, 4 \mathrm{H}, \mathrm{SCH}_{2} \mathrm{CH}_{2} \mathrm{~S}\right)$, $3.64\left(\mathrm{~s}, 4 \mathrm{H}\right.$, benzyl. $\left.\mathrm{CH}_{2}\right), 7.25(\mathrm{~m}, 8 \mathrm{H}$, aromat. H) - MS: $m / z$ $(\%)=398(100)\left[\mathrm{M}^{+\cdot}\right], 207(25), 178(17), 146(8), 132(29), 90(40)$. $\mathrm{C}_{21} \mathrm{H}_{22} \mathrm{~N}_{2} \mathrm{O}_{2} \mathrm{~S}_{2} \quad$ Ber. 398.1123 Gef. 398.1125 (MS) 
14,15-( Dimethylmalonyl)-2,6-dithia-14,15-diaza/7.2Jmetacyclophan (4b): Ausb. $398 \mathrm{mg}$ (45\%), Schmp. $106^{\circ} \mathrm{C}$ (Essigester), $R_{\mathrm{f}}=$ 0.65 (Petrolether/Essigester, 2:1). - ${ }^{1} \mathrm{H}-\mathrm{NMR}\left(80-\mathrm{MHz}, \mathrm{CDCl}_{3}\right)$ : $\delta=1.51\left(\mathrm{~s}, 6 \mathrm{H}, \mathrm{CH}_{3}\right), 2.16\left(\mathrm{~m}, 6 \mathrm{H}, \mathrm{S}\left[\mathrm{CH}_{2}\right]_{3} \mathrm{~S}\right), 3.57(\mathrm{~s}, 4 \mathrm{H}$, benzyl. $\left.\mathrm{CH}_{2}\right), 7.24\left(\mathrm{~m}, 8 \mathrm{H}\right.$, aromat. H). - MS: $m / z(\%)=412(100)\left[\mathrm{M}^{+\cdot}\right]$, 305 (64), 207 (34), 178 (25), 146 (13), 132 (23), 90 (40).

$\mathrm{C}_{22} \mathrm{H}_{24} \mathrm{~N}_{2} \mathrm{O}_{2} \mathrm{~S}_{2} \quad$ Ber. 412.1280 Gef. 412.1283 (MS)

15,16-(Dimethylmalonyl)-2,7-dithia-15,16-diaza/8.2Jmetacyclophan (4c): Ausb. $330 \mathrm{mg}$ (39\%), Schmp. $123^{\circ} \mathrm{C}$ (Essigester), $R_{\mathrm{f}}=$ 0.75 (Petrolether/Essigester, 2:1). - ${ }^{1} \mathrm{H}-\mathrm{NMR}\left(60 \mathrm{MHz}, \mathrm{CDCl}_{3}\right)$ : $\delta=1.20\left(\mathrm{~m}, 4 \mathrm{H}, \mathrm{CH}_{2}\left[\mathrm{CH}_{2}\right]_{2} \mathrm{CH}_{2}\right), 1.50\left(\mathrm{~s}, 6 \mathrm{H}, \mathrm{CH}_{3}\right), 1.98(\mathrm{~m}, 4 \mathrm{H}$, $\left.\mathrm{SCH}_{2}\right), 3.61$ (s, $4 \mathrm{H}$, benzyl. $\left.\mathrm{CH}_{2}\right), 7.20(\mathrm{~m}, 8 \mathrm{H}$, aromat. $\mathrm{H})$. - MS: $m / z(\%)=426(100)\left[\mathrm{M}^{+} \cdot\right], 330(21), 305(50), 207(23), 146(5)$, $132(10)$.

\section{$\mathrm{C}_{23} \mathrm{H}_{26} \mathrm{~N}_{2} \mathrm{O}_{2} \mathrm{~S}_{2} \quad$ Ber. 426.1436 Gef. 426.1434 (MS)}

17,18-( Dimethylmalonyl)-2,9-dithia-17,18-diaza(10.2/metacyclophan (4d): Ausb. $795 \mathrm{mg}\left(82 \%\right.$ ), Schmp. $128^{\circ} \mathrm{C}$ (Essigester), $R_{f}=$ 0.68 (Petrolether/Essigester, 2:1). - ${ }^{1} \mathrm{H}-\mathrm{NMR}\left(60 \mathrm{MHz}, \mathrm{CDCl}_{3}\right)$ : $\delta=1.25\left(\mathrm{~m}, 8 \mathrm{H}, \mathrm{CH}_{2}\left[\mathrm{CH}_{2}\right]_{4} \mathrm{CH}_{2}\right), 1.50\left(\mathrm{~s}, 6 \mathrm{H}, \mathrm{CH}_{3}\right), 2.22(\mathrm{~m}, 4 \mathrm{H}$, $\left.\mathrm{SCH}_{2}\right), 3.65\left(\mathrm{~s}, 4 \mathrm{H}\right.$, benzyl. $\left.\mathrm{CH}_{2}\right), 7.20(\mathrm{~m}, 8 \mathrm{H}$, aromat. $\mathrm{H})$. - MS: $m / z(\%)=454(83)\left[\mathrm{M}^{+\cdot}\right], 207(41), 147(38), 132(19), 55(100)$.

$\mathrm{C}_{25} \mathrm{H}_{30} \mathrm{~N}_{2} \mathrm{O}_{2} \mathrm{~S}_{2}$ Ber. 454.1749 Gef. 454.1750 (MS)

19,20-(Dimethylmalonyl)-2,11-dithia-19,20-diazal 12.2 Jmetacyclophan (4e): Ausb. $580 \mathrm{mg},(56 \%)$, Schmp. $114^{\circ} \mathrm{C}, R_{\mathrm{f}}=0.64$ (Petrolether/Essigester, 2:1). - ${ }^{1} \mathrm{H}-\mathrm{NMR}\left(300 \mathrm{MHz}, \mathrm{CDCl}_{3}\right): \delta=$ $1.30\left(\mathrm{~m}, 4 \mathrm{H}, \mathrm{CH}_{2}\left[\mathrm{CH}_{2}\right]_{2} \mathrm{CH}_{2}\right), 1.51\left(\mathrm{~s}, 6 \mathrm{H}, \mathrm{CH}_{3}\right), 1.65(\mathrm{~m}, 4 \mathrm{H}$, $\left.\mathrm{SCH}_{2} \mathrm{CH}_{2}\right), 2.29\left(\mathrm{t}, 4 \mathrm{H}, J=7 \mathrm{~Hz}, \mathrm{SCH}_{2} \mathrm{CH}_{2}\right), 3.66(\mathrm{~s}, 4 \mathrm{H}$, benzyl. $\left.\mathrm{CH}_{2}\right), 7.20(\mathrm{~m}, 8 \mathrm{H}$, aromat. $\mathrm{H})$. - MS: $m / z(\%)=482(100)\left[\mathrm{M}^{+\cdot}\right]$, 308 (37), 207 (42), 178 (42), 146 (17), 132 (41), 90 (25).

$\mathrm{C}_{27} \mathrm{H}_{34} \mathrm{~N}_{2} \mathrm{O}_{2} \mathrm{~S}_{2}$ Ber. 482.2062 Gef. 482.2061 (MS)

23,24-( Dimethylmalonyl-2,15-dithia-23,24-diazal 16.2 Jmetacyclophan (4f): Ausb. $739 \mathrm{mg}(64 \%)$, Schmp. $109^{\circ} \mathrm{C}, R_{\mathrm{f}}=0.59$ (Petrolether/Essigester, 2:1). - ${ }^{1} \mathrm{H}-\mathrm{NMR}\left(60 \mathrm{MHz}, \mathrm{CDCl}_{3}\right): \delta=1.17(\mathrm{~m}$, $\left.16 \mathrm{H}, \mathrm{CH}_{2}\left[\mathrm{CH}_{2}\right]_{8} \mathrm{CH}_{2}\right), 1.51\left(\mathrm{~s}, 6 \mathrm{H}, \mathrm{CH}_{3}\right), 1.58\left(\mathrm{~m}, 4 \mathrm{H}, \mathrm{SCH}_{2} \mathrm{CH}_{2}\right)$, $2.28\left(\mathrm{~m}, 4 \mathrm{H}, \mathrm{SCH} \mathrm{CH}_{2}\right), 3.69\left(\mathrm{~s}, 4 \mathrm{H}\right.$, benzyl. $\left.\mathrm{CH}_{2}\right), 7.20(\mathrm{~m}, 8 \mathrm{H}$, aromat. H). - MS: $m / z(\%)=538(100)\left[\mathrm{M}^{+} \cdot\right], 364(46), 307(15)$, $207(40), 178(13), 146(18), 132(45)$.

\section{$\mathrm{C}_{31} \mathrm{H}_{42} \mathrm{~N}_{2} \mathrm{~S}_{2} \mathrm{O}_{2} \quad$ Ber. 538.2688 Gef. 538.2684 (MS)}

Arbeitsvorschrift für die Abspaltung der Dimethylmalonyl-Schutzgruppe: $0.6-1.7 \mathrm{mmol} 4 \mathrm{a}-\mathrm{f}$ und $0.9 \mathrm{~g}(13 \mathrm{mmol}) \mathrm{NaOEt}$ werden in $50 \mathrm{ml}$ trockenem 1,2-Dimethoxyethan unter $\mathrm{N}_{2}$ bei Raumtemp. $12 \mathrm{~h}$ gerührt. Dann wird zur Trockene eingeengt, in Wasser aufgenommen, mit Essigester überschichtet und vorsichtig mit verd. $\mathrm{HCl}$ angesäuert, bis gerade eine Phasentrennung erfolgt. Es wird so lange mit Essigester extrahiert, bis die Extrakte farblos sind. Die vereinigten Extrakte werden mit Wasser gewaschen und mit $\mathrm{Na}_{2} \mathrm{SO}_{4}$ getrocknet. Die Reinigung erfolgt über eine Säule mit Kieselgel 60, Eluens Petrolether/Essigester (2:1).

2,5-Dithia-13,14-diaza/6.2/metacyclophan-13-en (5a): Eingesetzt $250 \mathrm{mg}(0.65 \mathrm{mmol}) 4 \mathrm{a}$; Ausb. $100 \mathrm{mg}(53 \%), R_{\mathrm{f}}=0.81$ (Petrolether/ Essigester, 2:1), Schmp. $117^{\circ} \mathrm{C}$ - - ${ }^{\mathrm{H}} \mathrm{H}-\mathrm{NMR}$ : siehe Tab. 2. - MS: $m / z(\%)=300(83)\left[\mathrm{M}^{+\cdot}\right], 211(18), 180(100), 165(32), 90(36), 89$ 131). $\mathrm{C}_{16} \mathrm{H}_{16} \mathrm{~N}_{2} \mathrm{~S}_{2}$ Ber. 300.0755 Gef. 300.0750 (MS)

2.6-Dithia-14,15-diaza[7.2]metacyclophan-14-en (5b): Eingesetzt $350 \mathrm{mg}(0.85 \mathrm{mmol}) 4 \mathrm{~b}$; Ausb. $170 \mathrm{mg}(63 \%) ; R_{\mathrm{f}}=0.67$ (cis-5b),
0.75 (trans-5b) (Petrolether/Essigester, 4:1), Schmp. $123^{\circ} \mathrm{C} .-{ }^{\mathrm{t}} \mathrm{H}$ NMR: siehe Tab. 2. - MS: $m / z(\%)=314(91)\left[\mathrm{M}^{+*}\right], 211$ (19), 180 (100), 165 (49), 121 (36), 90 (62), 89 (28).

\section{$\mathrm{C}_{17} \mathrm{H}_{18} \mathrm{~N}_{2} \mathrm{~S}_{2}$ Ber. 314.0911 Gef. 314.0910 (MS)}

2,7-Dithia-15,16-diaza/8.2]metacyclophan-15-en (5c): Eingesetzt $325 \mathrm{mg}(0.76 \mathrm{mmol}) 4 \mathrm{c}$; Ausb. $200 \mathrm{mg}(79 \%) ; R_{\mathrm{f}}=0.66($ cis-5c), 0.73 (trans-5c) (Petrolether/Essigester, 4:1), Schmp. $142^{\circ} \mathrm{C} .-{ }^{1} \mathrm{H}$ NMR: siehe Tab. 2. - MS: $m / z(\%)=328(87)\left[\mathrm{M}^{+\cdot}\right.$ ], $211(18)$, 180 (100), 165 (46), 121 (22), $90(78), 89$ (62).

\section{$\mathrm{C}_{18} \mathrm{H}_{20} \mathrm{~N}_{2} \mathrm{~S}_{2}$ Ber. 328.1053 Gef. 328.1060 (MS)}

2.9-Dithia-17,18-diaza/ 10.2Jmetacyclophan-17-en (5d): Eingesetzt $710 \mathrm{mg}(1.56 \mathrm{mmol}) 4 \mathrm{~d}$; Ausb. $418 \mathrm{mg}(75 \%) ; R_{\mathrm{f}}=0.68$ (cis-5d), 0.78 (trans-5d) (Petrolether/Essigester, 2:1), Schmp. $149^{\circ} \mathrm{C} .-{ }^{1} \mathrm{H}-$ NMR: siehe Tab. 2. - MS: $m / z(\%)=356(95)\left[\mathrm{M}^{+\cdot}\right], 211(18)$, $180(100), 165(26), 90(78), 89(43)$.

\section{$\mathrm{C}_{20} \mathrm{H}_{24} \mathrm{~N}_{2} \mathrm{~S}_{2}$ Ber. 356.1383 Gef. 356.1381 (MS)}

2,11-Dithia-19,20-diazal 12.2Jmetacyclophan-19-en (5e): Eingesetzt $550 \mathrm{mg}$ ( $1.14 \mathrm{mmol}) 4 \mathrm{e}$; Ausb. $290 \mathrm{mg}(66 \%), R_{\mathrm{f}}=0.63(\mathrm{Pe}-$ trolether/Essigester, 4:1), Schmp. $87 \mathrm{C}$ - ${ }^{1} \mathrm{H}-\mathrm{NMR}$ : siehe Tab. 2. - MS: $m / z(\%)=384(100)\left[\mathrm{M}^{+\cdot}\right], 211(17), 180(83), 165(30)$, $90(94), 89(39)$

\section{$\mathrm{C}_{22} \mathrm{H}_{28} \mathrm{~N}_{2} \mathrm{~S}_{2}$ Ber. 384.1694 Gef. 384.1694 (MS)}

2,15-Dithia-23,24-diaza/ 16.2/metacyclophan-23-en (5f): Eingesetzt $700 \mathrm{mg}$ (1.63 mmol) 4f; Ausb. $410 \mathrm{mg} \mathrm{(72 \% )} \mathrm{Öl,} R_{\mathrm{f}}=0.53$ (Petrolether/Essigester, 6:1). - ${ }^{1} \mathrm{H}-\mathrm{NMR}$ : siehe Tab. 2. - MS: $m / z(\%)=440(100)\left[\mathrm{M}^{+\cdot}\right], 211(15), 180(39), 165(16), 90(42), 89$ (12). $\mathrm{C}_{26} \mathrm{H}_{36} \mathrm{~N}_{2} \mathrm{~S}_{2} \quad$ Ber. 440.2320 Gef. 440.2323 (MS)

\section{CAS-Registry-Nummern}

1: 121012-94-0 / 2: 121012-95-1/3a: 540-63-6 / 3b: 109-80-8 / 3c: 1191-08-8 / 3d: 1191-43-1 / 3e: 1191-62-4/3f: 33528-63-1/4a: 121013-01-2 / 4b: 121029-40-1/4c: 121013-02-3/4d: 12101303-4 / 4e: 121013-04-5/4f: 121013-05-6/5a: 121012-96-2/5b (Isomer 1): 121012-97-3 / 5b (Isomer 2): 121054-71-5/5c: (Isomer 1): 121029-39-8 / 5c (Isomer 2): 121013-06-7 / 5d (Isomer 1): 121012-98-4 / 5d (Isomer 2): 121054-72-6 / 5e: 121012-99-5 / 5f: 121013-00-1 / Dimethylmalonsäuredichlorid: 5659-93-8 / m-Hydrazotoluol: $621-26-1$

1) U. Funke, H.-F. Grützmacher, Tetrahedron 43 (1987) 3787.

2) 2a) Neuere Ubersicht: F. Vögtle, Supramolekulare Chemie, Kap. 7, Teubner, Stuttgart 1989. - ${ }^{\text {b) }}$ H. Dürr, B. Ruge, Top. Curr. Chem. 66 (1976) 53. - ${ }^{2 c)}$ H. Rau, J. Photochem. 66 (1984) 221. 2d) S. Shinkai, T. Minami, Y. Kusano, O. Manabe, J. Am. Chem. Soc. 105 (1983) 1851. - ${ }^{2 e)}$ H. Rau, E. Lüddecke, J. Am. Chem. Soc. 104 (1982) 1616.

3) R. H. Mitchell in Cyclophanes (P. M. Keehn, S. M. Rosenfeld. Eds.), Bd. 1, Kap. 4, Academic Press Inc., London - New York 1983.

4) U. Dittrich, H.-F. Grützmacher, Chem. Ber. 118 (1985) 4404 , 4415.

5) J. Schmiegel, Diplomarbeit Univ. Bielefeld, 1986; zur Veröffentlichung vorbereitet.

6) W. Baker, J. F. W. McOmie, W. D. Ollis, J. Chem. Soc. 1951, 200.

7) 7a) J. J. Gajewski, K. E. Gilbert, $M M P M I$, QCPE Indiana 1986. - ${ }^{7 b)}$ R. M. Jarret, M. Saunders, STRPI, Yale Rapid Interactive Structure Input Program, Yale University 1983, modifiziert für MMPMI von J. J. Gajewski, K. E. Gilbert, Indiana 1986.

8) F. Vögtle, Chem.-Ztg. 96 (1972) 396. 